\title{
A Single Pipeline Embolization Device is Sufficient for Treatment of Intracranial Aneurysms
}

\author{
N. Chalouhi, S. Tjoumakaris, J.L.H. Phillips, R.M. Starke, D. Hasan, C. Wu, M. Zanaty, D. Kung, L.F. Gonzalez, R. Rosenwasser,
} and P. Jabbour

\begin{abstract}
BACKGROUND AND PURPOSE: The Pipeline Embolization Device has emerged as an important treatment option for intracranial aneurysms. The number of devices needed to treat an aneurysm is uncertain and is the subject of vigorous debate. The purpose of this study was to compare rates of complications, aneurysm occlusion, and outcome in patients treated with a single-versus-multiple Pipeline Embolization Devices.
\end{abstract}

MATERIAL AND METHODS: One hundred seventy-eight patients were treated with the Pipeline Embolization Device at our institution. Patients were divided into 2 groups: a single-device group $(n=126)$ and a multiple-device group $(n=52)$.

RESULTS: There was no statistically significant difference between the 2 groups with respect to baseline characteristics except for age and aneurysm size (higher with multiple Pipeline Embolization Devices). Complications occurred more frequently with multiple (15\%) versus a single device $(5 \%, P=.03)$. In multivariate analysis, the use of multiple devices independently predicted complications. A similar proportion of patients achieved adequate aneurysm obliteration at follow-up in the single-device (84\%) and the multiple-device groups (87\%, $P=.8$ ). In multivariate analysis, age and follow-up time predicted obliteration. At follow-up, a significantly higher proportion of patients treated with a single device (97\%) achieved a favorable outcome compared with those treated with multiple devices $(89 \%, P=.03)$. In multivariate analysis, there was a strong trend for the use of a single device to predict favorable outcomes $(P=.06)$.

CONCLUSIONS: Treatment with a single Pipeline Embolization Device provides similar occlusion rates with less complications and better overall outcomes. These findings suggest that a single Pipeline Embolization Device is sufficient for treatment of most intracranial aneurysms.

ABBREVIATIONS: $\mathrm{ICH}$ = intracerebral hemorrhage; $\mathrm{PED}=$ Pipeline Embolization Device

$\mathrm{T}$ he Pipeline Embolization Device (PED; Covidien, Irvine, California) is a dedicated flow diverter, which received US Food and Drug Administration approval in 2011 for the treatment of large and giant wide-neck aneurysms of the internal carotid artery. The device appears to be highly efficient in promoting durable aneurysm occlusion, and its safety profile has been shown to be favorable in several well-designed studies. ${ }^{1-3}$ In an increasing number of cerebrovascular centers, the PED has become a routine first-line option for unruptured intracranial aneurysms.

Received October 22, 2013; accepted after revision January 15, 2014

From the Department of Neurosurgery (N.C., S.T., J.L.H.P., R.M.S., C.W., M.Z., D.K., L.F.G., R.R., P.J.), Thomas Jefferson University and Jefferson Hospital for Neuroscience, Philadelphia, Pennsylvania; and Department of Neurosurgery (D.H.), University of lowa, lowa City, lowa.

Please address correspondence to Pascal M. Jabbour, MD, Division of Neurovascular Surgery and Endovascular Neurosurgery, Department of Neurological Surgery, Thomas Jefferson University Hospital, 901 Walnut St, 3rd Floor, Philadelphia PA 19107; e-mail: pascal.jabbour@jefferson.edu

http://dx.doi.org/10.3174/ajnr.A3957
The number of PEDs needed to treat an aneurysm is uncertain and is the subject of vigorous debate at present. ${ }^{4}$ The question is a crucial one and has potential implications for procedural cost, safety, and efficacy. To our knowledge, this is the first study to compare rates of complications, aneurysm occlusion, and outcome in patients treated with single-versus-multiple PEDs.

\section{MATERIALS AND METHODS Patient Population}

The study protocol was approved by the Thomas Jeffersion University Institutional Review Board. All patients with intracranial aneurysms treated with the PED at our institution between May 2011 and May 2013 were identified from a prospectively maintained data base. One hundred seventy-eight patients were treated during this period and constituted our study population.

The patient population was divided into 2 groups based on the number of PEDs deployed during initial aneurysm embolization. There were $126(70.8 \%)$ patients treated with a single device and $52(29.2 \%)$ patients treated with $>1$ device. The rates of compli- 
cations, aneurysm obliteration, retreatment, and clinical outcome were compared between the 2 groups. All symptomatic procedural complications were included. Delayed complications were also recorded through follow-up. Angiographic follow-up was scheduled at 3-6 months, 1 year, 2 years, and 5 years after treatment. Aneurysm occlusion at follow-up was categorized as complete/near-complete $(\geq 90 \%)$ or incomplete $(<90 \%)$. Clinical follow-up was performed at 1,3, and 6 months. Outcome was reported according to the modified Rankin Scale.

\section{Technique}

All patients received $75 \mathrm{mg} /$ day of clopidogrel (Plavix) and 81 $\mathrm{mg} /$ day of aspirin for 10 days before the procedure. Platelet function tests were performed on all patients by using aspirin and P2Y12 assays (VerifyNow; Accumetrics, San Diego, California). The procedure was performed only if the platelet inhibition level was $>30 \%$ but less $<90 \%$. Patients with inhibition $<30 \%$ were reloaded, and the assay was rechecked. Patients found to be poor responders to clopidogrel were then switched to prasugrel (40-mg loading dose followed by a $5-\mathrm{mg}$ daily maintenance dose). Patients with inhibition above $90 \%$ were admitted to the hospital, their procedure was canceled, and clopidogrel was held until the platelet inhibition level fell below 90\%. An initial heparin bolus was administered, and activated clotting time was maintained at 2-3 times the patient's baseline intraoperatively. Heparin was discontinued at the end of the procedure. Patients were maintained on dual antiplatelet therapy for at least 6 months after the procedure followed by aspirin indefinitely.

PEDs were generally deployed through a Marksman microcatheter (Covidien) by using a triaxial guide-catheter system to maximize support during forward loading of the system and to optimize stent opening and apposition. Inadequate vessel wall apposition was remedied with balloon angioplasty when needed. The number of devices deployed was dependent on the operator's preference and experience but was also based on subjective assessment of intra-aneurysmal stasis. When the landing zone was missed, further devices were placed to cover the aneurysm neck. In addition, we may tend to use $>1$ device specifically for fusiform aneurysms. In the basilar artery, whenever possible, we try to avoid using $>1$ device to preserve the patency of side branches. Placement of additional PEDs was considered at follow-up if the aneurysm remained unchanged or did not sufficiently decrease in size despite treatment.

\section{Statistical Analysis}

Data are presented as mean and range for continuous variables and as frequency for categoric variables. Analysis was performed by using an unpaired $t$ test, Wilcoxon rank sum test, $\chi^{2}$ test, and Fisher exact test as appropriate. Univariate analysis was used to test covariates predictive of the following dependent variables, each independently: complications, aneurysm obliteration at follow up, retreatment, and unfavorable outcome (mRS 3-6). Interaction and confounding were assessed through stratification and relevant expansion covariates. Factors predictive in univariate analysis $(P<.20)^{5}$ were entered into a multivariate logistic regression analysis. $P$ values $\leq .05$ were considered statistically signifi-
Table 1: Aneurysm locations

\begin{tabular}{lcc}
\multicolumn{1}{c}{ Aneurysm Location } & $\begin{array}{c}\text { 1 PED } \\
\text { (\%) }\end{array}$ & $\begin{array}{c}\text { Multiple } \\
\text { PEDs (\%) }\end{array}$ \\
\hline Carotid cavernous artery & $17(13.5)$ & $13(25)$ \\
Carotid ophthalmic artery & $53(42)$ & $19(36.5)$ \\
Paraclinoid artery & $36(28.6)$ & $7(13.5)$ \\
Middle cerebral artery & $4(3.1)$ & $5(9.6)$ \\
Basilar trunk & $3(2.4)$ & $2(3.8)$ \\
Posterior circulation & $8(6.4)$ & $4(7.7)$ \\
$\quad$ (other than basilar trunk) & & \\
Other & $5(4.0)$ & $2(3.9)$ \\
Total & 126 & 52 \\
\hline
\end{tabular}

cant. Statistical analysis performed with STATA 10.0 (StataCorp, College Station, Texas).

\section{RESULTS}

\section{Baseline Characteristics}

The proportion of female patients was $86 \%(108 / 126)$ in the single-PED group and $81 \%(42 / 52)$ in the multiple-PED group $(P=$ .4). Patients in the multiple-PED group were significantly older than those in the single-PED group (mean age, 61.1 years versus 54.2 years; $P=.002)$. Mean aneurysm size was also higher in the multiple-PED group (11.8 $\mathrm{mm}$ versus $9.2 \mathrm{~mm}, P=.02$ ), but the proportion of patients with large aneurysms did not differ significantly between the 2 groups (39\%, 49/126, with a single PED versus $52 \%, 27 / 52$, with multiple PEDs; $P=.2$ ). The proportion of aneurysms arising from the posterior circulation did not differ between the 2 groups $(8.7 \%, 11 / 126$, with a single PED versus $11.5 \%, 6 / 52$, with multiple PEDs; $P=.6$ ). Likewise, the proportion of aneurysms arising from the basilar trunk did not differ between the 2 groups $(2.4 \%, 3 / 126$, with a single PED versus $3.8 \%$, $2 / 52$, with multiple PEDs; $P=.8$ ). The distribution of aneurysm locations is detailed in Table 1. Fusiform or dissecting aneurysms accounted for $12.7 \%(16 / 126)$ and $23 \%(12 / 52)$ of aneurysms treated with a single and multiple PEDs, respectively $(P=.09)$. The proportion of previously treated aneurysms was comparable in those treated with single $(20.6 \%, 26 / 126)$ versus multiple devices $(19.2 \%, 10 / 52 ; P=.8)$.

Adjunctive coiling was undertaken in 11\% (14/126) of aneurysms treated with a single PED versus $5.8 \%$ (3/52) of those treated with multiple PEDs $(P=.3)$. Balloon angioplasty was necessary in $\approx 9.5 \%(12 / 126,5 / 52)$ of patients in either group $(P=.9)$. A single PED was used in $126(70.8 \%)$ patients; 2 PEDs, in $40(22.5 \%)$; 3 PEDs, in 7 (3.9\%); 4 PEDs, in $4(2.3 \%)$; and 5 PEDs, in $1(0.7 \%)$.

\section{Outcomes}

Complications occurred more frequently in those treated with multiple $(15 \%, 8 / 52)$ versus a single device $(5 \%, 7 / 126, P=.03)$. In the single-device group, there were 3 hemorrhagic complications ( 1 distal parenchymal hemorrhage, 1 spontaneous delayed aneurysm rupture after 4 months, and 1 aneurysm rupture due to proximal device migration causing a direct jet of blood against the dome) and 4 ischemic complications (1 associated with distal device migration in a patient with schizophrenia who was not compliant with his antiplatelet regimen). In the multiple-PED group, there were 4 hemorrhagic complications (all distal parenchymal 
Table 2: Aneurysm obliteration

\begin{tabular}{lccc}
\multicolumn{1}{c}{ Latest Occlusion } & $\begin{array}{c}\text { Single } \\
\text { PED (\%) }\end{array}$ & $\begin{array}{c}\text { Multiple } \\
\text { PED (\%) }\end{array}$ & $\begin{array}{c}\text { Total } \\
(\%)\end{array}$ \\
\hline Complete (100\%) & $58(68.2)$ & $28(70)$ & $86(68.8)$ \\
Near-complete (90\%-99\%) & $13(15.3)$ & $7(17.5)$ & $20(16)$ \\
Incomplete (<90\%) & $15(16.5)$ & $5(12.5)$ & $19(15.2)$ \\
Total & 85 & 40 & 125 \\
\hline
\end{tabular}

hemorrhages) and 4 ischemic complications. None of the hemorrhagic complications occurred in patients with middle cerebral artery aneurysms. The following factors were tested as predictors of complications: age, sex, aneurysm location, aneurysm size, aneurysm morphology, previous aneurysm treatment, number of PEDs used per aneurysm, adjunctive use of coils, and balloon angioplasty. In univariate analysis, predictors of complications were the following: 1$)$ increasing age $(P=.04) ; 2)$ increasing aneurysm size $(P=.004)$; and 3$)$ the use of $>1$ device $(P=.04)$. These factors were subsequently entered into a multivariate analysis. In multivariate analysis, the use of $>1$ device was the only statistically significant predictor of complications $(\mathrm{OR}=3.0 ; 95 \%$ CI, $1.1-8.8 ; P=.04)$.

Angiographic follow-up was available for 85 patients in the single-PED group and 40 patients in the multiple-PED group. Mean follow-up time was longer in the multiple- (8.9 months) than in the single-PED group (7.0 months, $P=.01)$. A similar proportion of patients achieved complete or near-complete aneurysm obliteration at follow-up in the single-device $(71 / 85,84 \%)$ and multiple-device $(35 / 40,87 \%, P=.8)$ groups (Table 2$)$. The following factors were tested as predictors of aneurysm obliteration: age, sex, aneurysm location, aneurysm size, aneurysm morphology, previous aneurysm treatment, number of PEDs used per aneurysm, adjunctive use of coils, balloon angioplasty, procedural complications, and angiographic follow-up time. In univariate analysis, the following factors were negative predictors of aneurysm obliteration $(<90 \%)$ : 1$)$ increasing age $(P=.1)$, and 2$)$ decreasing angiographic follow-up duration $(P=.09)$. In multivariate analysis, increasing age $(\mathrm{OR}=0.4 ; 95 \% \mathrm{CI}, 0.16-0.91$; $P=.03)$ and decreasing angiographic follow-up duration $(\mathrm{OR}=$ $0.9 ; 95 \% \mathrm{CI}, 0.8-0.99 ; P=.04$ ) were independent negative predictors of aneurysm obliteration. The number of devices was not a predictive factor, even after controlling for these variables.

Retreatment was necessary in a similar proportion of patients after embolization with single $(6 \%, 5 / 85)$ or multiple devices $(7.5 \%, 3 / 40, P=.8)$. In-stent stenosis was noted in $\approx 5 \%(4 / 85$, $2 / 40)$ of patients in each group $(P=.95)$.

Clinical follow-up was available for 125 patients in the singlePED group and 52 patients in the multiple-PED group. At the latest follow-up, a significantly higher proportion of patients treated with a single device $(97 \%, 121 / 125)$ achieved a favorable outcome ( $\mathrm{mRS} 0-2$ ) compared with those treated with $>1$ device $(89 \%, 46 / 52, P=.03)$. In the single-PED group, 3 of the 4 patients with an unfavorable outcome sustained a procedural complication and 1 was disabled at baseline. In the multiple-PED group, 4 of the 6 patients with an unfavorable outcome sustained a complication and 1 was severely disabled before PED treatment. The same factors as those used for occlusion were tested as predictors of clinical outcome. In univariate analysis, the following factors were predictors of unfavorable outcome (mRS $>2)$ : 1 ) increasing aneurysm size $(P>.001)$, and 2$)$ the use $>1$ device $(P=.03)$. In multivariate analysis, increasing aneurysm size $(\mathrm{OR}=1.1 ; 95 \%$ CI, $1.06-1.6 ; P=.001)$ was an independent predictive factor, and there was a strong trend toward the use of $>1$ device to predict unfavorable outcome $(\mathrm{OR}=3.5 ; 95 \% \mathrm{CI}, 0.9-13 ; P=.06)$.

\section{DISCUSSION}

Flow diversion is now a well-established treatment for intracranial aneurysms. ${ }^{2,6-10}$ Flow diverters, most typically the PED, have proved to be more efficient than and at least as safe as traditional embolization strategies. ${ }^{6,11,12}$ Lanzino et al ${ }^{13}$ reported a significantly higher rate of complete occlusion in patients with the PED $(76 \%)$ than in patients $(21 \%)$ treated with coils with a similar rate of morbidity. Likewise, Chalouhi et $\mathrm{al}^{2}$ compared the safety and efficacy of the 2 techniques in unruptured, large, and giant $(\geq 10$ $\mathrm{mm}$ ) aneurysms, reporting a similar complication rate $(7.5 \%)$ and a higher aneurysm occlusion rate ( $86 \%$ versus $41 \%$ ) with flow diversion. They concluded that the PED is a preferred treatment option for large unruptured saccular aneurysms. The Pipeline for Uncoilable or Failed Aneurysms study is a recently published prospective, international multicenter series that included large and giant, wide-neck aneurysms arising from the internal carotid artery. ${ }^{1}$ The results of this study confirmed the safety $(5.6 \%$ rate of major ipsilateral stroke or neurologic death) and high efficacy of the PED (73.6\% rate of complete occlusion at day 180 without major stenosis of the parent vessel) in the most complex aneurysms.

Aside from thromboembolic complications, which may occur in any neuroendovascular procedure, treatment with flow diverters carries the risk of specific complications not encountered with standard embolization techniques. Distal intracerebral hemorrhage $(\mathrm{ICH})$ is a dreaded complication of flow diversion, and its incidence is reported to be approximately $1 \%-2 \% .{ }^{14,15}$ The mechanism of ICH after flow diversion may involve hemorrhagic conversion of ischemic infarcts in the setting of dual antiplatelet therapy, modification of intracranial blood pressure in the distal territories, and intraprocedural embolization of foreign material. $^{2,16,17}$ Delayed aneurysm rupture after flow diversion occurs in approximately $1.0 \%$ of patients, and its mechanism may involve sudden hemodynamic changes in intra-aneurysmal flow patterns or aneurysm wall weakening due to inflammation and proteolytic enzymes within the developing red thrombus. ${ }^{17}$ Some have suggested using intraluminal coils or steroids to attenuate the effects of intra-aneurysmal thrombosis, but whether this strategy can reduce the risk of aneurysm rupture remains to be seen. Last, device migration has recently been recognized as a potential, severe complication of flow diversion that can be associated with hemorrhagic or thromboembolic events. ${ }^{18}$ Migration may occur proximally or distally and is related to the mismatch in arterial diameter between inflow and outflow vessels and to stretching of the device. ${ }^{19}$

The present study was undertaken to address a pressing and crucial question that has been recently debated in the neurointerventional community. Specifically, we aimed to determine whether treatment with a single PED is sufficient for treatment of intracranial aneurysms. Although there might not be a categoric 
answer to this question, this study provides strong evidence supporting the use of only a single PED in the initial treatment of aneurysms. As such, the complication rate was thrice as high in the multiple-device group (15\%) compared with the single-device group ( $5 \%)$. Moreover, the use of $>1$ device was a strong independent predictor of complications with an odds ratio as high as 3. We believe that this is explained by a higher propensity for thromboembolic complications with the use of multiple PEDs, which are highly thrombogenic constructs with high metal surface area coverage. Because ICH may be secondary to ischemic lesions, hemorrhagic events are more likely to occur when multiple devices are used as well. In addition, it is well known that placement of the second and third PED is usually more technically complex, given the presence of the previously deployed device. The difficulty of deploying PEDs over previous stents has been highlighted in a recent article. ${ }^{10}$ Although one may naturally tend to think that placing additional devices provides further flow diversion and better angiographic results, the present study demonstrates similar occlusion rates at follow-up with the use of a single (84\%) versus multiple devices (87\%). This crucial finding suggests that a single device provides enough flow diversion for occlusion of most aneurysms and that placement of additional devices will only add morbidity, cost, and radiation exposure. Accordingly, we believe that complete intra-aneurysmal stasis during initial embolization is not required for ultimate aneurysm thrombosis and should not be considered the end point of the procedure. Using multiple devices did not decrease the rate of retreatment; this result may suggest that aneurysms that fail flow diversion have an inherent tendency to do so regardless of the number of devices used. The senior author's strategy entails treating most aneurysms with a single PED initially and reserving further device deployment only for those aneurysms that remain unchanged or do not sufficiently decrease in size at the 3- to 6-month follow-up. This strategy avoids the unnecessary use of additional devices and is undoubtedly an important factor in procedural safety. The trend toward better clinical outcomes in the single-device group further supports this strategy. Potential exceptions include fusiform aneurysms, ruptured aneurysms in which immediate occlusion is warranted, and cases in which the initial device has not provided adequate neck coverage or vessel reconstruction. Using multiple PEDs may increase the stability of the construct and prevent device migration. ${ }^{20}$ Also, there were no aneurysm ruptures in the multiple-device group. The 2 aneurysm ruptures that occurred in the single-device group may suggest that both could have been prevented with a multiple-device construct from the outset.

Although the studied question has not been previously addressed, the literature may suggest a similar trend. In the German study by Fischer et al, ${ }^{21}$ in which multiple devices were used in as many as $66 \%$ of cases (median, 3 ), major complications occurred in $6 \%$ of patients. Likewise, the major complication rate was $8.5 \%$ in the US multicenter series in which 2.12 PEDs were used on average per aneurysm. ${ }^{22}$ In the Canadian experience, O'Kelly et $\mathrm{al}^{23}$ used $>1$ device in almost half of the patients and experienced a combined morbidity and mortality rate of $10.7 \%$ (6.3\% mortality, $4.4 \%$ morbidity). In contrast, Lylyk et $\mathrm{al}^{24}$ used a single PED in $70 \%$ of aneurysms and reported a $0 \%$ rate of morbidity and mor- tality. Likewise, Saatci et al ${ }^{14}$ used only 1.3 PEDs on average per aneurysm ( 1 device in $\approx 70 \%$ ) and noted a combined morbiditymortality rate as low as $1.5 \%$. Thus, the use of multiple devices may be associated with higher complication rates and worse clinical outcomes, as corroborated in the present report.

This study is limited by its overall retrospective design, though the safety data on the PED are prospectively recorded at our institution. This study reports the experience of a single cerebrovascular center; thus, results may not be entirely generalizable. Although the 2 groups were well-matched with regard to most baseline characteristics, the older age, larger aneurysm size, and higher proportion of fusiform aneurysms (though not significantly) in the multiple-PED group may have favored the singledevice group. Fusiform aneurysms are higher risk lesions with poorer outcomes across all treatment methods. Some may argue that the higher occurrence of intraparenchymal hemorrhages in the multiple-device group may be related to more challenging cases with a higher risk of wire perforations and more manipulation, causing more vessel stretching and tearing. However, in contrast to coiling, complication and recurrence rates with the PED are not affected by aneurysm size. ${ }^{19,25}$ This outcome limits the effect of this factor on the comparative analysis. In addition, the shorter follow-up time in the single-PED group has favored the multiple-PED group because the occlusion rate of aneurysms treated with flow diverters increases with time. ${ }^{3,14}$ A multivariate analysis was performed to control for those differences, and it identified treatment with multiple devices as an independent predictor of complications and poor outcome (strong trend).

\section{CONCLUSIONS}

The results of this study show that treatment with a single PED is associated with fewer complications, better functional outcomes, and similar occlusion rates compared with multiple PEDs. These results suggest that a single $\mathrm{PED}$ is sufficient for treatment of most intracranial aneurysms. Placement of additional devices may only add morbidity, cost, and radiation exposure.

In the era of flow diverters, ongoing trials should not only confirm the noninferiority of flow diversion to traditional embolization techniques (which is already known from several welldesigned studies) but should also investigate ways to further improve the procedural safety and efficacy of flow diverters.

Disclosures: Stavropoula Tjoumakaris—UNRELATED: Consultancy: Stryker Neurovascular. Pascal Jabbour-UNRELATED: Consultancy: Covidien.

\section{REFERENCES}

1. Becske T, Kallmes DF, Saatci I, et al. Pipeline for Uncoilable or Failed Aneurysms: results from a multicenter clinical trial. Radiology 2013;267:858-68

2. Chalouhi N, Tjoumakaris S, Starke RM, et al. Comparison of flow diversion and coiling in large unruptured intracranial saccular aneurysms. Stroke 2013;44:2150-54

3. Yu SC, Kwok CK, Cheng PW, et al. Intracranial aneurysms: midterm outcome of Pipeline embolization device: a prospective study in 143 patients with 178 aneurysms. Radiology 2012;265:893-901

4. Tse MM, Yan B, Dowling RJ, et al. Current status of Pipeline embolization device in the treatment of intracranial aneurysms: a review. World Neurosurg 2013;80:829-35

5. Altman DG. Practical Statistics for Medical Research. Boca Raton, Florida: Chapman \& Hall/CRC; 1999 
6. Yavuz K, Geyik S, Saatci I, et al. Endovascular treatment of middle cerebral artery aneurysms with flow modification with the use of the Pipeline embolization device. AJNR Am J Neuroradiol 2014;35:529-35

7. McAuliffe $\mathrm{W}$, Wycoco $\mathrm{V}$, Rice $\mathrm{H}$, et al. Immediate and midterm results following treatment of unruptured intracranial aneurysms with the Pipeline embolization device. AJNR Am J Neuroradiol 2012;33:164-70

8. Chalouhi N, Chitale R, Starke RM, et al. Treatment of recurrent intracranial aneurysms with the Pipeline embolization device. J Neurointerv Surg 2014;6:19-23

9. Chalouhi N, Starke RM, Yang S, et al. Extending the indications of flow diversion to small, unruptured, saccular aneurysms of the anterior circulation. Stroke 2014;45:54-58

10. Chalouhi N, Tjoumakaris S, Dumont AS, et al. Treatment of posterior circulation aneurysms with the Pipeline embolization device. Neurosurgery 2013;72:883-89

11. Crobeddu E, Lanzino G, Kallmes DF, et al. Marked decrease in coil and stent utilization following introduction of flow diversion technology. J Neurointerv Surg 2013;5:351-53

12. Brinjikji W, Murad MH, Lanzino G, et al. Endovascular treatment of intracranial aneurysms with flow diverters: a meta-analysis. Stroke 2013;44:442-47

13. Lanzino G, Crobeddu E, Cloft HJ, et al. Efficacy and safety of flow diversion for paraclinoid aneurysms: a matched-pair analysis compared with standard endovascular approaches. AJNR Am J Neuroradiol 2012;33:2158-61

14. Saatci I, Yavuz K, Ozer C, et al. Treatment of intracranial aneurysms using the Pipeline flow-diverter embolization device: a single-center experience with long-term follow-up results. AJNR Am J Neuroradiol 2012;33:1436-46

15. Leung GK, Tsang AC, Lui WM. Pipeline embolization device for intracranial aneurysm: a systematic review. Clin Neuroradiol 2012;22:295-303
16. Deshmukh V, Hu YC, McDougall CG, et al. Histopathological assessment of delayed ipsilateral parenchymal hemorrhages after the treatment of paraclinoid aneurysms with the Pipeline embolization device. Neurosurgery 2012;71:E551-52

17. Pierot L, Wakhloo AK. Endovascular treatment of intracranial aneurysms: current status. Stroke 2013;44:2046-54

18. Chalouhi N, Satti SR, Tjoumakaris S, et al. Delayed migration of a Pipeline embolization device. Neurosurgery 2013;72(2 suppl operative):ons229-34, discussion ons 234

19. Jabbour P, Chalouhi N, Tjoumakaris S, et al. The Pipeline embolization device: learning curve and predictors of complications and aneurysm obliteration. Neurosurgery 2013;73:113-20, discussion 120

20. Chalouhi N, Tjoumakaris SI, Gonzalez LF, et al. Spontaneous delayed migration/shortening of the Pipeline embolization device: report of 5 cases. AJNR Am J Neuroradiol 2013;34:2326-30

21. Fischer S, Vajda Z, Aguilar Perez M, et al. Pipeline embolization device (PED) for neurovascular reconstruction: initial experience in the treatment of 101 intracranial aneurysms and dissections. Neuroradiology 2012;54:369-82

22. Kan P, Siddiqui AH, Veznedaroglu E, et al. Early postmarket results after treatment of intercrainal aneurysms with the Pipeline embolization device: a US multicenter experience. Neurosurgery 2012; 71:1080-87

23. O'Kelly CJ, Spears J, Chow M, et al. Canadian experience with the Pipeline embolization device for repair of unruptured intracranial aneurysms. AJNR Am J Neuroradiol 2013;34:381-87

24. Lylyk P, Miranda C, Ceratto R, et al. Curative endovascular reconstruction of cerebral aneurysms with the Pipeline embolization device: the Buenos Aires experience. Neurosurgery 2009;64:632-42, discussion 642-43, quiz N6

25. Chalouhi N, Tjoumakaris S, Gonzalez LF, et al. Coiling of large and giant aneurysms: complications and long-term results of 334 cases. AJNR Am J Neuroradiol 2014;35:546-52 